\title{
Ureterocele found incidentally on focused assessment with sonography for trauma (FAST) exam
}

\author{
Adam Ash $^{1} \cdot$ Tanya Bajaj $^{1}$ Christopher Raio ${ }^{1}$
}

Received: 22 September 2015/Accepted: 8 December 2015/Published online: 28 December 2015 (C) SIMI 2015

\section{Introduction}

We present a case of a 33-year-old man with a ureterocele found incidentally on FAST examination during a trauma evaluation. This rare finding is important to recognize because it may be indicative of a duplicated collecting system or may contribute to renal insufficiency due to reflux. Generally, the evaluation requires additional imaging studies and urology consultation.

\section{Case}

A 33-year-old man presented to the emergency department (ED) as a level one trauma after being struck by a car while changing a flat tire on the side of the highway. He complained of right hip pain, was unable to walk, but denied any other injuries. Physical examination revealed a distressed, but well appearing man with normal vital signs and tenderness to palpation over his right hip. Although his abdomen was non-tender, a FAST exam was performed due to the mechanism of injury, and revealed the following image of the bladder (Fig. 1a). No Foley catheter had been placed in this patient.

\section{Discussion}

A ureterocele is a congenital cystic outpouching of the distal portion of the ureter into the bladder that arises as a result of abnormal embryogenesis, although its exact etiology is unknown. Its incidence is between 1:5000 and $1: 12,000$, symptoms vary, and range from none at all to life-threatening urosepsis [1]. The majority of ureteroceles $(80-90 \%)$ is associated with a duplicated collecting system, and is discovered early in life in young children presenting with urinary tract infections. 10-20\%, however, exist as simple ureteroceles and cause no symptoms [2]. These are frequently discovered incidentally while obtaining imaging for unrelated complaints. These cases may be managed with observation alone, although definitive imaging is required to exclude the presence of a duplicating collecting system is these patients. The patient in our case received a CT scan of the abdomen and pelvis that demonstrated a normal genitourinary system. CT imaging, while important for the diagnosis of a duplicated collecting system, is less sensitive for the diagnosis of ureterocele than ultrasound. This is especially true when the bladder is empty, causing compression of the ureterocele.
Adam Ash

adamash4@hotmail.com; adamash4@gmail.com

1 Department of Emergency Medicine, North Shore University Hospital, 300 Community Drive, Manhasset, NY 11030, USA 

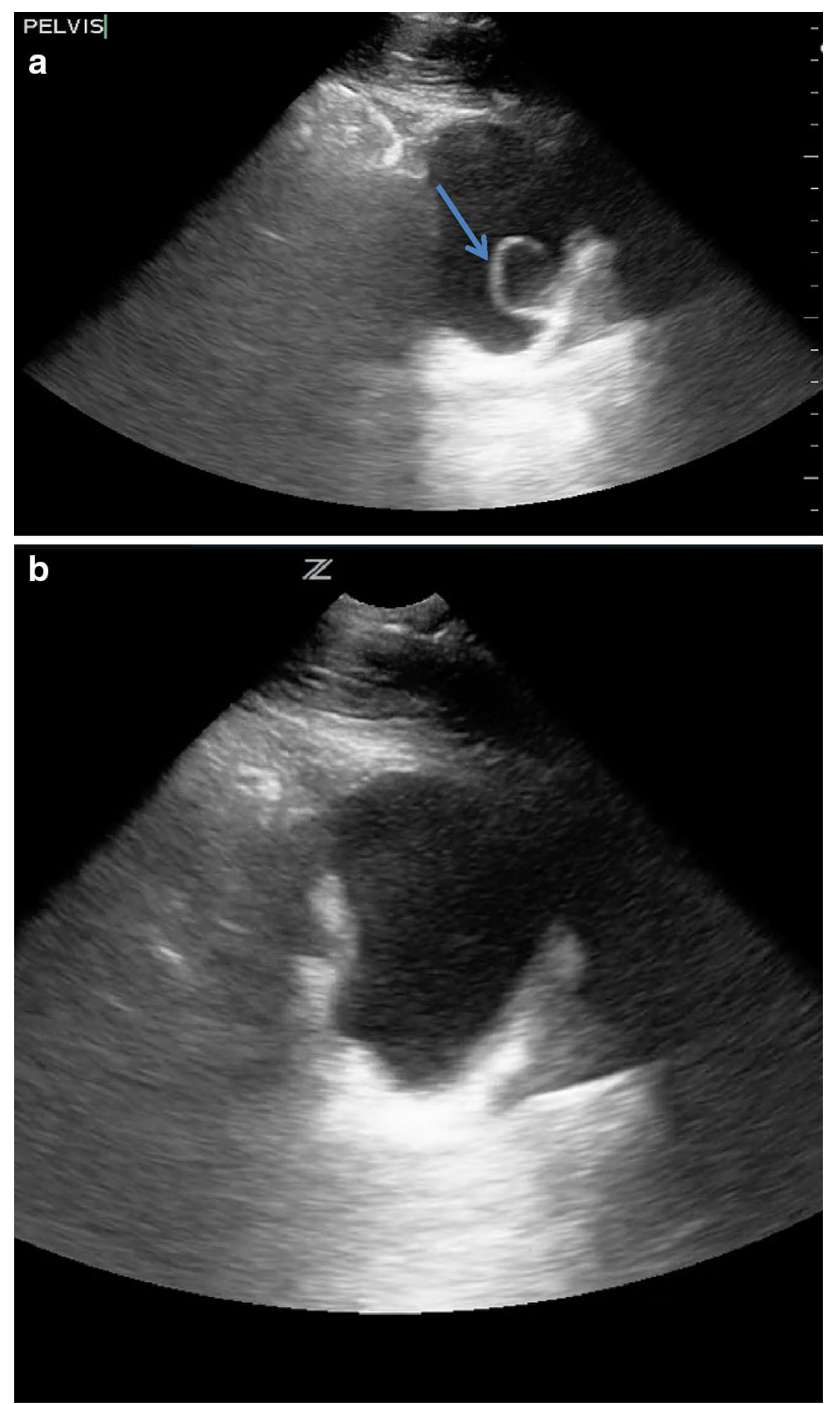

Fig. 1 a Cystic outpouching of the distal ureter (arrow) consistent with ureterocele. b Normal sagittal view of the bladder

\section{Compliance with ethical standards}

Conflict of interest Dr. Raio is an industry consultant fo Zonare, an ultrasound machine company.

Statement of human and animal rights All procedures performed in studies involving human participants were in accordance with the ethical standards of the institutional research committee and with the 1964 Helsinki declaration and its later amendments or comparable ethical standards.

Informed consent For this type of study, formal consent is not required.

\section{References}

1. Ayers E (2006) Incidental finding of bilateral ureteroceles. J Diagn Med Sonogr 22(2):123-126

2. Conlin MJ (1995) Current management of ureteroceles. Urology 45(3):357-362 